Etienne Gayat

Romain Pirracchio

Matthieu Resche-Rigon

Alexandre Mebazaa

Jean-Yves Mary

Raphaël Porcher

\section{Reply to Ferrer et al.: propensity scores in intensive care literature}

Accepted: 14 February 2011

Published online: 25 February 2011

(C) Copyright jointly held by Springer and ESICM 2011

This reply refers to the comment available at: doi:10.1007/s00134-011-2190-8.

Dear Editor,

We thank Ferrer et al. [1] for their critical reading of our review. Concerning their first comment regarding the Wisnivesky et al. [2] article, the reference was found on the PubMed database in 2009 as an e-publication. This explains why it was included in our review.

With regard to the Ferrer et al. [3] article, despite the double-checked research in PubMed in January 2010, we missed it and did not include it in our review. We apologize for this oversight. New analysis, including the additional paper, was performed and led to very minimal changes (Table 1).

Table 1 Characteristics of propensity score development of the three techniques (including the Ferrer et al. article [3])

\begin{tabular}{|c|c|c|c|c|}
\hline & All & Matching & Adjustment & Stratification \\
\hline No. of articles & $48(100)$ & $26(54)$ & $10(21)$ & $12(25)$ \\
\hline No. of patients & $\begin{array}{l}2,199 \\
{[502-5,385]}\end{array}$ & $\begin{array}{l}2,273 \\
{[586-9,340]}\end{array}$ & 640 & $\begin{array}{l}3,668 \\
{[824-5,838]}\end{array}$ \\
\hline \multicolumn{5}{|c|}{$\begin{array}{l}\text { Covariates included in the } \\
\text { propensity score model }\end{array}$} \\
\hline Number & $16[9-22]$ & 18 [14-32] & $10[5-16]$ & $12[8-13]$ \\
\hline $\begin{array}{l}\text { No. of treated patients } \\
\text { per covariate } \\
\text { included in the model }\end{array}$ & 20 [10-69] & $20[10-57]$ & $15[5-31]$ & 25 [16-151] \\
\hline Event/variable ratio $<10$ & $11(31)$ & $6(33)$ & $3(33)$ & $2(22)$ \\
\hline Names precised & 37 (79) & $18(72)$ & $10(91)$ & $8(80)$ \\
\hline Choice discussed & $26(54)$ & $12(46)$ & $6(55)$ & $7(70)$ \\
\hline \multicolumn{5}{|l|}{ Type of endpoint } \\
\hline Binary endpoint & $34(71)$ & $18(69)$ & $9(82)$ & $6(60)$ \\
\hline Censored endpoint & $11(23)$ & $6(23)$ & $2(18)$ & $3(30)$ \\
\hline Continuous endpoint & $3(6)$ & $2(8)$ & $0(0)$ & $1(10)$ \\
\hline
\end{tabular}

\section{References}

1. Ferrer R, Suarez D, Artigas A (2011) Propensity scores in intensive care literature. Intensive Care Med. doi: 10.1007/s00134-011-2190-8

2. Wisnivesky JP, Halm E, Bonomi M, Powell C, Bagiella E (2010)

Effectiveness of radiation therapy for elderly patients with unresected stage I and II non-small cell lung cancer. Am J Respir Crit Care Med 181:264-269

3. Ferrer R, Artigas A, Suarez D, Palencia E, Levy MM, Arenzana A, Perez XL, Sirvent JM (2009) Effectiveness of treatments for severe sepsis: a prospective, multicenter, observational study. Am J Respir Crit Care Med 180:861-866
E. Gayat $(\bowtie) \cdot R$. Pirracchio •

M. Resche-Rigon · J.-Y. Mary ·

R. Porcher

Clinical Epidemiology and Biostatistics, INSERM U717, University Paris 7, Paris, France

e-mail: etienne.gayat@1rb.aphp.fr

E. Gayat - R. Pirracchio - A. Mebazaa Department of Anesthesiology and Intensive Care, Lariboisière Hospital, University Paris 7, Paris, France

A. Mebazaa

INSERM U942, Paris, France 